\title{
Optimization of Milling Conditions by Using Particle Swarm Optimization Technique: A Review
}

\author{
Sagar Bahirje ${ }^{\# 1}$, Prof.Vishvajeet Potdar ${ }^{\# 2,}$ \\ ${ }^{2} P G$ Student of Manufacturing \& processing engineering, ${ }^{2}$ Vice principal \& Professor of Mechanical Engineering Department \\ ${ }^{1,2}$ A. G.Patil Institute of Technology Solapur \\ Solapur University \\ Maharashtra-413006 India
}

\begin{abstract}
- a review of publications associated with the optimization of milling conditions by particle swarm analysis method. Milling is the most common form of machining, a material removal process, which can create a variety of features on a part by cutting away the unwanted material In order to optimize the cutting conditions, the empirical relationships between input and output variables should be established in order to predict the output. Optimization of these predictive models helps us to select appropriate input variables for achieving the best output performance. In this review paper the study is covered regarding the optimization of different input parameters and results are analyzed.
\end{abstract}

Keywords - Milling, Particle swarm optimization, Process parameter optimization

\section{INTRODUCTION}

Milling is one of the progressive technologies which has wide range of application in industries and other related areas. Milling like any metal cutting operation is used with an objective of optimizing milling conditions at micro level and economic performance at macro level. In addition to milling parameters, modern manufacturers do not want any compromise on the achievement of high quality, dimensional accuracy, high production rate, minimum wear on the cutting tools, cost saving and increase of the performance of the product with minimum environmental hazards. Milling is a cutting procedure dependent of a number of variables. These variables are dependent from each other in consequence, if we change one variable, the others change too. Optimization techniques can be applied to the CNC milling program to improve cutting conditions, improve end finishing, reduce tool wear and reduce the stress on the tool, the machine and the machined part. Process optimization means the resources which are utilizing the process should be used effectively and efficiently at minimum cost \& maximum output. In optimization, we focus on different parameters which govern the process. In present scenario, it is a matter of great concern in industry to achieve a good quality product at minimum cost.

In the early 1990's Soft computing became a formal computer science area of study. Earlier computational approaches and methods could model and precisely analyse only relatively simple systems. More complex systems arising in machining, biology, medicine and similar fields often remained unsolvable to conventional mathematical and analytical methods. It should be pointed out that simplicity and complexity of systems are relative, and many conventional mathematical models have been both challenging and very productive. Soft computing deals with imprecision, uncertainty, partial truth and approximation to achieve tractability, robustness and low solution cost. Components of soft computing include Neural networks (NN), perceptron's, Fuzzy systems (FS), Evolutionary computation (EC) which include Evolutionary algorithms, genetic algorithms (GA), Hybrid (neuro - fuzzy, pseudo outerproduct-based fuzzy neural network and Swarm intelligence (Particle swarm optimization - PSO, ant colony optimization ACO). Above all are the optimization techniques to improve cutting conditions of machining operations.

\section{PARTICLE SWARM OPTIMIZATION TECHNIQUE}

Particle swarm optimization is used to optimize the predicted models for machining. Particle swarm optimization is one of the soft computing method which is extensively used in research work. PSO was developed in 1995 by James Kennedy (social-psychologist) and Russell Eberhart (electrical engineer).[1] It uses a number of agents (particles) that constitute a swarm moving around in the search space looking for the best solution. Each particle is treated as a Point in a Ndimensional space which adjusts its "flying" according to its own flying experience as well as the flying experience of other particles.it is a relatively new technique for optimization of continuous nonlinear functions. It is easy to implement in a few line of computer code. It requires only primitive mathematical operators so is computationally inexpensive in terms of both memory requirement and speed. PSO is an evolutionary computation technique and has merits of both Genetic Algorithm and evolution strategies GA is only helpful to solve combinatorial optimization problem on the other hand PSO effectively applied in both continuous optimization problem \& combinatorial optimization problem. 


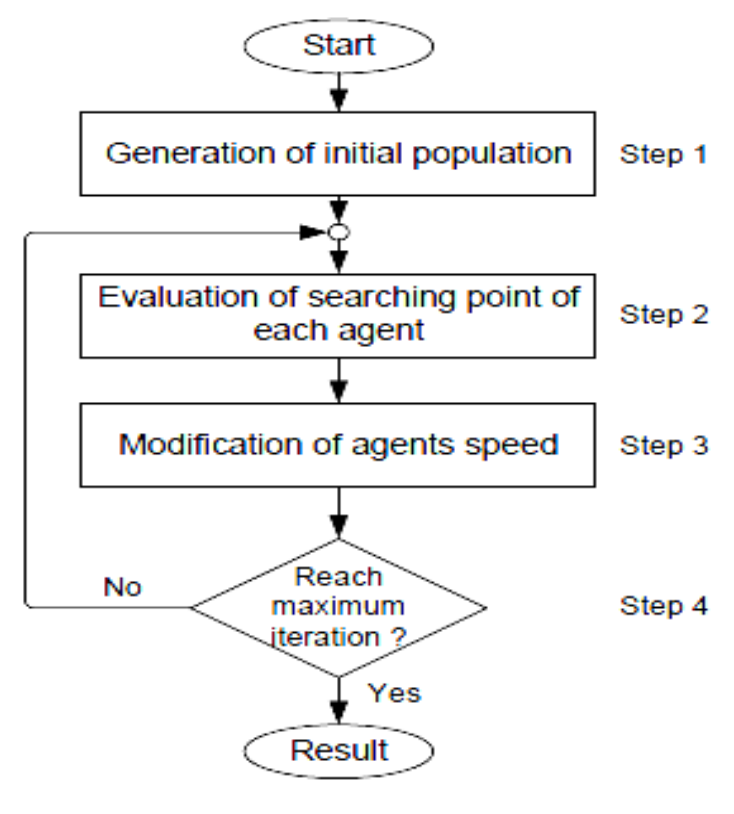

Figure 1.The general algorithm of PSO strategy [1]

Fig. 1 shows the general flow of PSO optimization technique in which first we have to generate the initial population for searching of best element. After solving the initial population equations we will get best search points in the population at step 2. And we have to replace the search space elements with optimized elements which will modify the search speed at step 3.if our optimization iteration reaches the maximum number to get best feasible solution then only we will get optimized results otherwise we have to go to step 2 again to evaluate searching point of each agent. After knowing the mathematical equations for milling machining parameters we can optimize different input parameters such as Process Kinematics, Cutting Fluid, Depth of Cut, Feed Rate, and Step over, Tool Angle, Cutting Speed. Etc. and output parameters can be Material removal rate, Tool life, Productivity, Machining time, cycle time reduction, Machining cost etc.

\section{LITERATURE REVIEW}

Kennedy and Eberhart [1](1995) developed a populationbased stochastic optimization technique known as Particle swarm optimization The PSO belongs to the class of direct search methods used to find an optimal solution to an objective function (fitness function) in a search space. Direct search methods are usually derivative-free, meaning that they depend only on the evaluation of the objective function. The particle swarm optimization algorithm is simple, in the sense that even the basic form of the algorithm shows results and it can be implemented very fast and is inspired by the social behaviour of bird flocking or fish schooling.

Marko Reibenschuh, Franc Cus, Uros Zuperl [2] has studied different optimization methods, used for optimizing the cutting conditions during milling \& also a part of using soft computer techniques and PSO and GA algorithm are applied to the CNC milling program to improve cutting conditions, improve end finishing, reduce tool wear and reduce the stress on the tool, the machine and the machined part. They observed that in process control procedures with the help of optimization tools we can reduce production time, improve end finishing by raising the quality and in the same time produce more parts. Also the process control procedures are very important, to assure proper function of the machining centre.

Azlon zain, Haron and sharif [3] observed the effect of different parameter like cutting speed, feed and rake angle in surface roughness. They compared the result of regression modeling and genetic algorithm. H.ganesan \& g.mohankumar, k.ganesan, k.ramesh kumar [4] studied the optimal machining parameters for continuous profile machining are determined with respect to the minimum production time, subject to a set of practical constraints, cutting force, power and dimensional accuracy and surface finish. Due to complexity of this machining optimization problem, a genetic algorithm (GA) and Particle Swarm Optimization (PSO) are applied to resolve the problem and the results obtained from GA and PSO are compared. has observed that many constraints were taken to account in order to make the model more realistic. A model of the process has been formulated with non-traditional algorithms; GA and PSO have been employed to find the optimal machining parameters for the continuous profile. PSO produces better results. Using this technique machining time can be further minimized.

Meenu \& anuj kumar sehgal [5] has experimented Particle Swarm Optimization (PSO), an evolutionary technique is used to optimize the machining parameters in $\mathrm{CNC}$ end milling of Ferritic-Pearlitic Ductile Iron Grade 80-55-06 with the objective of minimizing the surface roughness. Two methods, response surface methodology (RSM) and the Particle Swarm Optimization (PSO) are used to optimize the machining parameter i.e. surface roughness. The response surface modelling has shown the feasibility of predicting optimum response easily and successfully at the factor level settings for the surface roughness value to be $1.43 \mu \mathrm{m}$ with mean $\%$ error of $5.5 \%$ in comparison to confirmation experimental values. On the other hand the model prediction based on particle swarm optimization is found outperforming the RSM. The main advantages of the PSO algorithm are summarized as: simple concept, easy implementation, robustness to control parameters and computational efficiency when compared with mathematical algorithm and other heuristic optimization techniques

Vikas Pare, Geeta Agnihotri \& C.M. Krishna[6] has observed in order to optimize the surface finish, the empirical relationships between input and output variables should be established in order to predict the output. Optimization of these predictive models helps them to select appropriate input variables for achieving the best output performance. \& Particle swarm optimization technique is used for finding the optimum set of values of input variables and the results are compared with those obtained by GA optimization, and they found there is lot of scope for application of particle swarm optimization (PSO) for machining kind of problems by taking more number of input variables.

Saravanan R Baskar N., Asokan P., and Prabhaharan, G.[7] showed significant improvement in conventional milling process optimization by using various non-conventional optimization techniques such as PSO \& They compared their 
results with the results obtained from hand books. Alauddin, M., El Baradie, M. A., and Hashmi, M. S.J., [8] used cutting speed, feed and depth of cut which are three important parameters to predict the surface roughness by using Particle swarm optimization. Tandon, V.,El-Mounayri. H. and Kishawy, H., [9] optimized feed and speed CNC milling process. By using PSO technique .Rao, R. V., Savsani, V. J., and Vakharia, D. P., [10] introduced a new optimization method known as Teaching - Learning Based Optimization (TLBO).This algorithm has not only solved many bench mark design problems and given effective and efficient result compared the result with other non-traditional optimization techniques such as PSO, ACO, SA, GA, etc.

Bharti R.S et al.[11] suggested the machining performance viz. machining time and surface roughness in CNC machining after conducting experiments on brass, aluminium, copper, and mild steel. PSO was used to find the optimal machining parameters for minimizing machining time subjected to desired surface roughness. Physical constraints for both experiment and theoretical approach were cutting speed, feed, depth of cut, and surface roughness. It was observed that the machining time and surface roughness based on PSO are nearly same as that of the values obtained based on confirmation experiments; hence, it was found that PSO is capable of selecting appropriate machining parameters for turning operation. Since few years the non- traditional optimization techniques such as Ant Colony Optimization, Particle Swarm Optimization (PSO) along with the artificial neural network and Genetic Algorithm are gaining the great importance, especially in machining investigations. So the necessity to explore the additional robust techniques for prediction of desired surface roughness under milling machining is evident.

In the research by Prakasvudhisarn et al. [12], process parameters of CNC end milling were selected such as feed rate, spindle speed, and depth of cut to find the minimum surface roughness. Support vector machine (SVM) was proposed to capture characteristics of roughness and its factors. PSO technique is then employed to find the combination of optimal process parameters. The results showed that cooperation between both techniques can achieve the desired surface roughness and also maximize productivity simultaneously

J.G. Lil et al. [13] used particle swarm optimization (PSO) for cutting parameters optimization (CPO). The fundamental principle of PSO was introduced; then, the algorithm for PSO application in cutting parameters optimization was developed; thirdly, cutting experiments without and with optimized cutting parameters were conducted to demonstrate the effectiveness of optimization, respectively. The results showed the improvement in machining process.

Zarei, O., Fesangharyb, M., Farshia, B.,JaliliSaffarb, R., Razfarb, M.R., [14] proposed a harmony search (HS) algorithm to estimate the optimum cutting parameters for multi-pass face-milling process to minimize total production cost. Mainly four cutting parameters namely cutting speed, feed depth of cut/pass and number of passes are considered in their work. Harmony search algorithm has given significant improvement in result as compare to GA. A new technique has been proposed by Huang et al. [15] by using the combination of wavelet neural network (WNN) algorithm and modified PSO for solving tool wear detection and estimation. By using the Daubechies-wavelet, the cutting power signal is decomposed into approximation and details. The energy and square-error of the signals in the detail levels is used as characters which indicating tool wear, the characters are input to the trained WNN to estimate the tool wear. The results of the experiments were compared with BP neutral network, conventional WNN and GA-based WNN. The results showed a faster convergence and more accurate estimation of tool wear.

Khan M.A et al. [16] has prescribed the two different evolutionary algorithm-based neural network models developed to optimize the unit production cost. The hybrid neural network models proposed were genetic algorithmbased neural network (GA-NN) model and particle swarm optimization based neural network (PSO-NN) model. The objective considered was the minimization of unit production cost subjected to various machine constraints. An orthogonal design and analysis of variance was employed to determine the effective cutting parameters that affect the tool life. The GA-NN and PSO-NN models were compared for their performance. Optimal cutting conditions obtained with the PSO-NN model was found best possible compromise compared with the GA-NN model during machining GFRP composite using alumina cutting tool.

\section{CONCLUSIONS}

We can observe that particle swarm optimization has lot of scope for optimization problems by taking more number of input variables. We can take more number of input parameters to study different type of output parameter as well as combination of parameters. With the help of optimization tools like PSO we can reduce production time, improve end finishing by raising the quality and in the same time produce more parts. PSO has been basically developed for continuous optimization problem. PSO can be an efficient optimization tool for solving nonlinear continuous optimization problems, combinatorial optimization problems, and mixed-integer nonlinear optimization problem. To model the machining process, several important operational constraints have been considered. These constraints were taken to account in order to make the model more realistic. A model of the process has been formulated with non-traditional algorithms; GA and PSO have been employed to find the optimal machining parameters for the continuous profile. PSO produces better results. Using this technique machining time can be further minimized we can use this PSO technique for micro grinding, micro milling, micro drilling and laser application to optimize the output parameters effectively and PSO will help industrialist to find optimum values for input parameters to reduce cycle time as well as operational cost.

\section{REFERENCES}

[1] Kennedy, J., \& Eberhart, R. (1995). Particle swarm optimization. Proceedings of the IEEE International Conference on Neural Networks (ICNN'95), Perth, Australia. 
[2] Marko Reibenschuh F.Cus and Zuperl, "Particle swarm intelligence based optimization of high speed End milling."Vol3 pp383-393

[3] Haron and Shariff (2010) "Application of GA to optimize cutting condition for minimizing surface roughness in end milling machining process",Vol37,pp 4650-4659

[4] H.ganesan \& g.mohankumar, k.ganesan, k.ramesh kumar "optimization of machining parameters in turning process using genetic algorithm and particle swarm optimization with experimental verification" ISSN : 0975-5462 Vol. 3 No. 2 Feb 2011

[5] Meenu \& anuj kumar sehgal" surface roughness optimization by respose Surface methodology and particle swarm optimization" ISSN : 0975-5462 Vol. 5 No.07 July 2013

[6] Vikas Pare, Geeta Agnihotri \& C.M. krishna I "Optimization of Cutting Conditions in End Milling Process with the Approach of Particle Swarm Optimization" ssn no. 2231 -6477, volume-1, issue-2, 2011

[7] Baskar, N., Asokan, P., Saravanan, R. and Prabhaharan, G., (2004) "Optimization of machining parameters for milling operations using Non-conventional methods", Int J Adv Manuf. Technol., Vol. 25, pp. 1078-1088

[8] Alauddin, M., El Baradie, M. A., and Hashmi, M. S. J., (1995) "Computer-aided analysis of a surface-roughness model for end milling”, J. Mater. Process. Technol, Vol. 55, pp. 123-127.

[9] Tandon, V., El-Mounayri. H. and Kishawy, H., (2002), "NC end milling optimization using evolutionary computation", International Journal of Machine Tools \& Manufacture, Vol. 42, pp. 595-605.

[10] Rao, R. V., Savsani, V. J., and Vakharia, D. P., (2011), "Teacher learning - based optimization: A novel method for constrained mechanical design optimization problems", Computer - Aided Design, Vol. 43, pp. 303-315

[11] Bharathi, R. S., \& Baskar, N. (2011). Particle swarm optimization technique for determining optimal machining parameters of different work piece materials in turning operation. International Journal of Advanced Manufacturing Technology, 54(5-8), 445-463.

[12] Prakasvudhisarn, C., Kunnapapdeelert, S., \& Yenradee, P., 2009. Optimal cutting condition determination for desired surface roughness in end milling, International Journal of Advanced Manufacturing Technology, 41(5-6), 440-451

[13] J.G. Li1, Y.X. Yao1, D. Gao1, C.Q. Liu1 and Z.J. Yuan, 2008. Cutting Parameters Optimization by Using Particle Swarm Optimization (PSO), Applied Mechanics and Materials Vols. 10-12 pp. 879-883

[14] Zarei, O., Fesangharyb, M., Farshia, B., JaliliSaffarb, R., Razfarb, M.R., (2008), "Optimization of multi-pass face-milling via harmony search algorithm” J. Mater. Process. Tech., Vol. 5, pp. 1-7.

[15] Huang, H., Li, A., \& Lin, X. 2007. Applications of PSO-based wavelet neural network in tool wear monitoring. Proceedings of the IEEE international conference on automation and logistics pp. 2813-2817.

[16] M. Adam Khan \& A. Senthil Kumar \& A. Poomari, 2011. A hybrid algorithm to optimize cutting parameter for machining GFRP composite using alumina cutting tools. Int J Adv Manuf Technol DOI 10.1007/s00170-011-3553-6. 